\title{
Reconstruction of the depletion layer in MOSFET by genetic algorithms
}

\author{
El Yazidi Youness, Ellabib Abdellatif \\ Laboratory of Applied Mathematics and Computer Science, \\ Faculty of Science and Technology, Cady Ayyad University, \\ Avenue Abdelkrim El Khattabi B. P., 549, Marrakesh, Morocco
}

(Received 22 February 2020; Revised 18 March 2020; Accepted 20 March 2020)

\begin{abstract}
In this work, the MOSFET device is considered. The carrier densities in the MOSFET are modeled by the drift-diffusion equation. We manipulate the formulas of the charge density at the equilibrium in order to derive a simple Poisson's or Laplace's equation. To formulate a shape optimization problem, we have defined a cost functional. The existence of an optimal solution is proved. To solve the involved optimization problem, we have designed a numerical approach based on the finite element method combined with the genetic algorithm. Several numerical examples are established to prove the validity of the proposed approach.
\end{abstract}

Keywords: semiconductor, shape optimization, finite element, genetic algorithm.

2010 MSC: 49Q10, 65L60, 90C59

DOI: $10.23939 / \mathrm{mmc} 2020.01 .096$

\section{Introduction}

Inverse boundary identification problems are ubiquitous in medicine, engineering, industry, and other fields [1-3]. This kind of problem consists in finding an unknown boundary defined as a part of the domain boundary's or a boundary which separates two regions, that is called a free interface. Likely in the framework of semiconductor industry the depletion layer is assumed to be a free boundary, it is the interface which separates two regions, the depletion and conductivity regions. In this paper, we study the reconstruction of the depletion layer in the MOSFET (Metal-Oxide-Semiconductor Field-Effect Transistor) device [4].

To solve a free boundary problem, we pass through two phases, the first consists in defining a shape functional that can help to transform the free boundary problem into a shape optimization problem, then prove the existence of an optimal solution. The second phase implies the design of a numerical method in order to approximate the free boundary or the free interface numerically.

Several numerical schemes to solve this kind of problem have been proposed, most of them are gradient based methods, such as the famous conjugate gradient method combined with some discretization scheme, such as the work of Abouchabaka et al. [1] they use the boundary elements method combined with gradient method to solve an optimization problem in MESFET device. The work of Zhang P. [5], deals with the application of the finite element combined with a gradient method to solve an inverse scattering problem. In this work we put the light on the heuristic methods, they are considered as feasible strategies to solve a given optimization problem. Gradient based methods have a major inconvenience, which is the need to evaluate the gradient of the cost functional, in the case of shape optimization it concerns the computation of the shape derivative that can be evaluated by solving two subproblems named the sensitivity and the adjoint problems. There comes the simplicity of Heuristic methods, they consist of finding the optimal solution in a population of candidate solutions, basing on the value of the cost functional that depends only on the solution of the state problem.

In this work, to solve the involved shape optimization problem we propose the combined finite element method [6] with the genetic algorithms [7]. The search of the optimal depletion layer is performed by the genetic algorithm process, that consists in moving an initial population of candidate 
solution to a new one based on the genetic operations, selection, mutation, and crossover. The optimal solution is obtained after exceeding a maximal generation or satisfying the stopping criteria.

This work is organized as follows, in the next section 2 we introduce the MOSFET and its mathematical model. In section 3 we introduce a cost functional to formulate the shape optimization problem. Section 4 is dedicated to describe the proposed scheme. In section 5, we establish some numerical examples to show the validity of the two approaches to approximate the depletion layer in MOSFET.

\section{Problem formulation}

The Metal Oxide Semiconductor Field Effect Transistor abbreviated by MOSFET is an important semiconductor device used mainly in the fabrication of microwaves, for its property such as the low noise signal amplifier [4]. MOSFET is composed from a drain, source and a gate terminals (Fig. 1). There is two working mode's of the MOSFET, the first one is the depletion mode, it is realized when the applied voltage to the gate terminal is zero, we say that the MOSFET is on. The other one is the enhancement mode, where the MOSFET is off, it is realized when we apply a negative voltage to the gate terminal.

The domain of the MOSFET is $\Omega$, we assume that $\partial \Omega$ is smooth. $C$ and $D$ are the conductivity and depletion regions respectively. We set $\Gamma_{D}=\Gamma_{2} \cup \Gamma_{3} \cup \Gamma_{4}$ and $\Gamma_{N}=\partial \Omega \backslash \Gamma_{D}$. where $\Gamma_{2}, \Gamma_{3}$ and $\Gamma_{4}$ are the source, the gate and the drain terminals, respectively. The depletion layer is denoted by $\Gamma$. The carriers density in the MOSFET can be described in the domain of MOSFET $\Omega$ by the following equation:

$$
\begin{aligned}
& -\varepsilon \Delta u=q(n-p-m), \\
& J_{n}=q\left(D_{n} \nabla n+\mu_{n} n \nabla u\right), \\
& J_{p}=-q\left(D_{p} \nabla p+\mu_{p} p \nabla u\right), \\
& \operatorname{div}\left(J_{n}\right)=0 \quad \operatorname{div}\left(J_{p}\right)=0,
\end{aligned}
$$

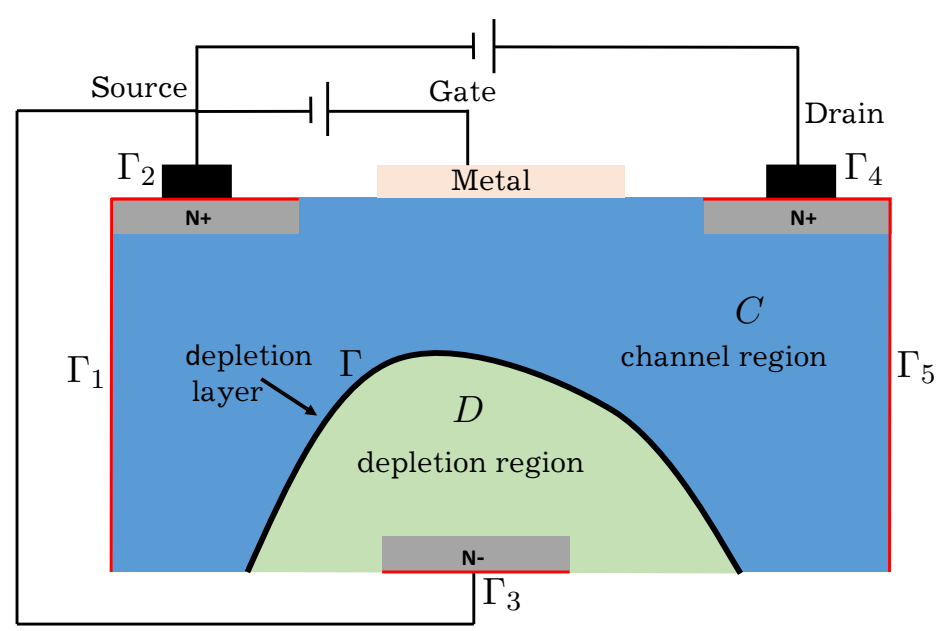

Fig. 1. Geometry of MOSFET.

$q$ is the elementary charge and $\varepsilon$ is the materials permittivity, they are also called the physical constants. $n, p$ and $m$ are the electrons, holes densities and the doping profile, respectively. $J_{n}$ and $J_{p}$ are the current densities. The variable $u$ stands for the electrostatic potential.

We add to the system (1) the next boundary conditions

$$
\begin{aligned}
& n=n_{d} \quad p=p_{d} \quad u=u_{d} \text { on } \Gamma_{D} \\
& \frac{\partial n}{\partial \nu}=\frac{\partial p}{\partial \nu}=\frac{\partial u}{\partial \nu}=0 \text { on } \Gamma_{N} .
\end{aligned}
$$

As we want to identify the depletion layer, we first need to simplify the previous expression using some relations on the charge density in the semiconductor at the equilibrium stage. In the equilibrium, inside the depletion region there are no mobile charges means that the ionized donor density equals the donor density $N$,

$$
n-p-m=N,
$$

and the conductivity region is neutral, means that

$$
n-p-m=0
$$

Mathematical Modeling and Computing, Vol. 7, No. 1, pp. 96-103 (2020) 
The above equations can be simplified as follows [4]:

$$
\left\{\begin{array}{rlll}
-\Delta u=0 & \text { on } & C, \\
-\Delta u=\zeta & \text { on } & & D, \\
\frac{\partial u}{\partial \nu}=0 & \text { on } & & \partial \Omega \cap \Gamma_{N}, \\
u=u_{d} & \text { on } & & \partial \Omega \cap \Gamma_{D},
\end{array}\right.
$$

where $u_{d}$ equal $V^{+}$on $\Gamma_{1}, V^{-}$on $\Gamma_{3}$ and 0 on $\Gamma_{5}$. And $\zeta=N / \varepsilon$. The equation (3) can be transformed into the following system (4)-(5):

$$
\begin{gathered}
\left\{\begin{array}{rll}
-\Delta u_{1}=0 & \text { on } & C, \\
\frac{\partial u_{1}}{\partial \nu}=0 & \text { on } & \partial C \cap \Gamma_{N}, \\
u_{1}=u_{d} & \text { on } & \partial C \cap \Gamma_{D},
\end{array}\right. \\
\left\{\begin{array}{rll}
-\Delta u_{2}=\zeta & \text { on } & D, \\
\frac{\partial u_{2}}{\partial \nu}=0 & \text { on } & \partial D \cap \Gamma_{N}, \\
u_{2}=u_{d} & \text { on } & \partial D \cap \Gamma_{D} .
\end{array}\right.
\end{gathered}
$$

The potential $u$ is assumed to be continuous on the depletion layer $\Gamma$, in order to guaranty the equivalence between equation (3) and the system (4)-(5), means that

$$
u_{1}=u_{2} \quad \text { and } \quad \frac{\partial u_{2}}{\partial \nu}=\frac{\partial u_{1}}{\partial \nu} \quad \text { on } \quad \Gamma .
$$

The unknown in (4)-(6) is the potential $u$ and the depletion layer $\Gamma$, in the next section we design the shape identification problem in order to determine $u$ and $\Gamma$.

\section{Shape identification problem}

First, let the functional space be: $H_{\Gamma_{D}}^{1}(\Omega)=\left\{u \in H^{1}(\Omega), u=0\right.$ on $\left.\Gamma_{D}\right\}$, where $H^{1}(\Omega)$ is the standard Sobolev space. It is obvious that $\Gamma_{D} \subseteq \partial \Omega$. The trace operator from $H^{1}(\Omega)$ in $H^{\frac{1}{2}}\left(\Gamma_{D}\right)$ is surjectif, then there exists $U \in H^{1}(\Omega)$ such $U=u_{d}$ on $\Gamma_{D}$, then the weak formulation of system (4)-(5) can be written as:

$$
\begin{aligned}
& \left\{\begin{array}{l}
\text { Find } u_{1} \in H_{\Gamma_{D}}^{1}(C) \text { such: } \\
\int_{C} \nabla u_{1}(x) \nabla v(x) d x=-\int_{C} \nabla U(x) \nabla v(x) d x, \quad \forall v \in H_{\Gamma_{D}}^{1}(C) .
\end{array}\right. \\
& \left\{\begin{array}{l}
\text { Find } u_{2} \in H_{\Gamma_{D}}^{1}(D) \text { such: } \\
\int_{D} \nabla u_{2}(x) \nabla v(x) d x=\int_{D} \zeta v(x) d x-\int_{D} \nabla U(x) \nabla v(x) d x, \quad \forall v \in H_{\Gamma_{D}}^{1}(D) .
\end{array}\right.
\end{aligned}
$$

Now, we assume that the free layer $\Gamma$ is parametrized by:

$$
\Gamma(\varphi)=\{(x, \varphi(x)) / x \in[0, L] \text { and } 0 \leqslant \varphi(x) \leqslant H\},
$$

$\varphi$ is the function characterizing the free boundary $\Gamma$. Then $\theta_{a d}$ the set of admissible layers is given by:

$$
\begin{aligned}
\theta_{a d}=\left\{\Gamma(\varphi) / \varphi \in \mathcal{C}([0, H]), \exists L_{0}>0,\left|\varphi\left(x_{1}\right)-\varphi\left(x_{2}\right)\right| \leqslant L_{0}\left|x_{1}-x_{2}\right|,\right. \\
\left.0 \leqslant \varphi\left(x_{1}\right) \leqslant H \forall x_{1}, x_{2} \in[0, H] \text { and } \varphi=0 \text { on }\left[0, a_{\Gamma}\right] \cup\left[b_{\Gamma}, L\right]\right\} .
\end{aligned}
$$


We note that for any admissible depletion layer $\Gamma \in \theta_{a d}$, the variational problems (7) and (8) admit unique solutions, the proof is based on the Lax-Milgram theorem [8].

Now we consider the following cost functional:

$$
J(\Gamma, u)=\frac{1}{2} \int_{\Gamma}\left(u_{1}-u_{2}\right)^{2} d x+\rho \operatorname{meas}(\Gamma),
$$

the first term in $J$ is to satisfy the continuity of the potential $u$ on the depletion layer, means that we must have $u_{1}=u_{2}$ on $\Gamma$ at convergence. The second term is a regularization term added to ensure the uniqueness of the optimal solution, defined as the measure of $\Gamma$ :

$$
\operatorname{meas}(\Gamma)=\int_{\Gamma} 1 d s
$$

$\rho$ is a penalty parameter. Let $\mathcal{F}$ be the following space:

$$
\mathcal{F}=\left\{(\Gamma, u), \Gamma \in \theta_{a d}, u=\left(u_{1}, u_{2}\right) ; \quad u_{1} \text { solution of }(7) \text { and } u_{2} \text { solution of }(8)\right\} .
$$

Finally, the shape identification problem can be given by the following:

$$
\left\{\begin{array}{l}
\text { Find }\left(\Gamma^{\star}, u^{\star}\right) \operatorname{such}: \\
\left(\Gamma^{\star}, u^{\star}\right)=\underset{(\Gamma, u) \in \mathcal{F}}{\operatorname{argmin}} J(\Gamma, u) .
\end{array}\right.
$$

Theorem 1. The problem (10) admits at least one solution in $\mathcal{F}$.

The proof of this theorem requires the compactness of the space $\mathcal{F}$ and the lower semi-continuity of functional $J$.

Proposition 1. The space $\mathcal{F}$ is compact.

Proof. First we demonstrate that $\theta_{a d}$ is compact. Let $\left(\Gamma^{(n)}\right)_{n}=\left(\Gamma\left(\varphi_{n}\right)\right)_{n}$ be sequence lives in $\theta_{a d}$, using the Ascoli-Arzela theorem, we deduce that $\left(\Gamma^{(n)}\right)_{n}$ converge to $\varphi$ in terms of subsequence. Since $\varphi_{n}=0$ on $\left[0, a_{n}\right] \cap\left[b_{n}, L\right]$ we can easily derive the existence $a$ and $b$ such that $a_{n} \longrightarrow a$ and $b_{n} \longrightarrow b$ uniformly in $[0, L]$, in addition we have $\varphi=0$ on $[0, a] \cap[b, L]$. Hence $\theta_{a d}$ is compact.

Let now $\left(u^{(n)}\right)$ solution sequence of (3). We note that a solution of $(3)$ is bounded in $H^{1}(\Omega)$ independently of $\Gamma$ [9], then $\left(u^{(n)}\right)$ is bounded, therefore we can extract a subsequence denoted again $\left(u^{(n)}\right)$ that converges to $u$ weakly in $H^{1}(\Omega)$. Similarly to [9], we can show that $u$ is a solution of (3) and the convergence of $\left(u^{(n)}\right)$ to $u$ is strong.

Lemma 2. The functional $J$ is lower semicontinuous on $\mathcal{F}$.

Proof. Let $\left(\Gamma^{(n)}, u^{(n)}\right)$ be a sequence in $\mathcal{F}$ that converges to $(\Gamma, u)$. Since we have proved the strong convergence of $\left(u^{n}\right)$ to $u$, then we have

$$
\lim _{n \rightarrow \infty} \int_{\Gamma^{(n)}}\left[u_{1}^{(n)}-u_{2}^{(n)}\right]^{2} d x=\int_{\Gamma}\left(u_{1}-u_{2}\right)^{2} d x
$$

On the other hand [8], we have

$$
\operatorname{meas}(\Gamma)=\int_{\Gamma} 1 d s \leqslant \liminf _{n \rightarrow \infty} \int_{\Gamma^{(n)}} 1 d s=\liminf _{n \rightarrow \infty} \operatorname{meas}\left(\Gamma^{(n)}\right) .
$$

Therefore,

$$
J(\Gamma, u) \leqslant \liminf _{n \rightarrow \infty} J\left(\Gamma^{(n)}, u^{(n)}\right),
$$

which prove the lower-semi continuity of $J$. 
As we mentioned in the introduction, to solve this shape identification problem, we propose an approach designed by a combination of the genetic algorithm with finite element method. The next section will concentrate on the description of this approach.

\section{The proposed algorithm}

Genetic algorithms (GA) [7,10] become a feasible strategy to solve a wide classes of optimization problems. GA works on a population of candidate solutions, the candidate solutions or the individuals should be represented by a binary or a float code, for the problem at hand the float representation can be implemented easily, in contrast the binary representation will cost a huge time from run to run. A new generation is created after applying the three genetic operators, selection, crossover, and mutation.

Selection: it may be the most important step in a genetic algorithm, it leads us to know which individuals will survive to be parents to create the future generation. Several selection operators exist,e.g. the roulette wheel selection, stochastic universal selection, and the tournament selection. In this application we use the tournament selection which takes a chosen number of individuals for a battle, the winners will be the parents for the next meeting.

Crossover: this genetic operator combines the information of two parents to produce new offspring or children. There are different crossover schemes such, one-point crossover, multi-point crossover, the random barycentric crossover, the crossover is applied with the probability $p_{c}$. In this work, we use the random barycentric crossover, from two parents $X_{1}, X_{2}$ and a weight $\alpha$ chosen randomly within the range $[0,1]$, it produces two new children: $Y_{1}=\alpha X_{1}+(1-\alpha) X_{2}$, and $Y_{2}=\alpha X_{2}+(1-\alpha) X_{1}$.

Mutation: it performers diversity in the population by changing one or more genes in an individual radically, for the binary mutation it reverses 0 to 1 or 1 to 0 . In our case, its desirable to use the nonuniform mutation or the Gaussian mutation. The mutation is applied with a probability $p_{m}$, as in nature the mutation is rare, therefore this probability should be small.

In order to simplify the task, we use the bézier curve [11] to parameterize our free boundaries, which means we will give just some control points in place of giving the entire coordinates of the boundary. As advantages of the bézier curves is that the curve is a continuous and infinitely differentiable curve, and it is bounded by the convex envelope formed by the control points. We note that the individuals will be vectors of control points, then we use the Casteljau algorithm [11] to convert each individual to a curve.

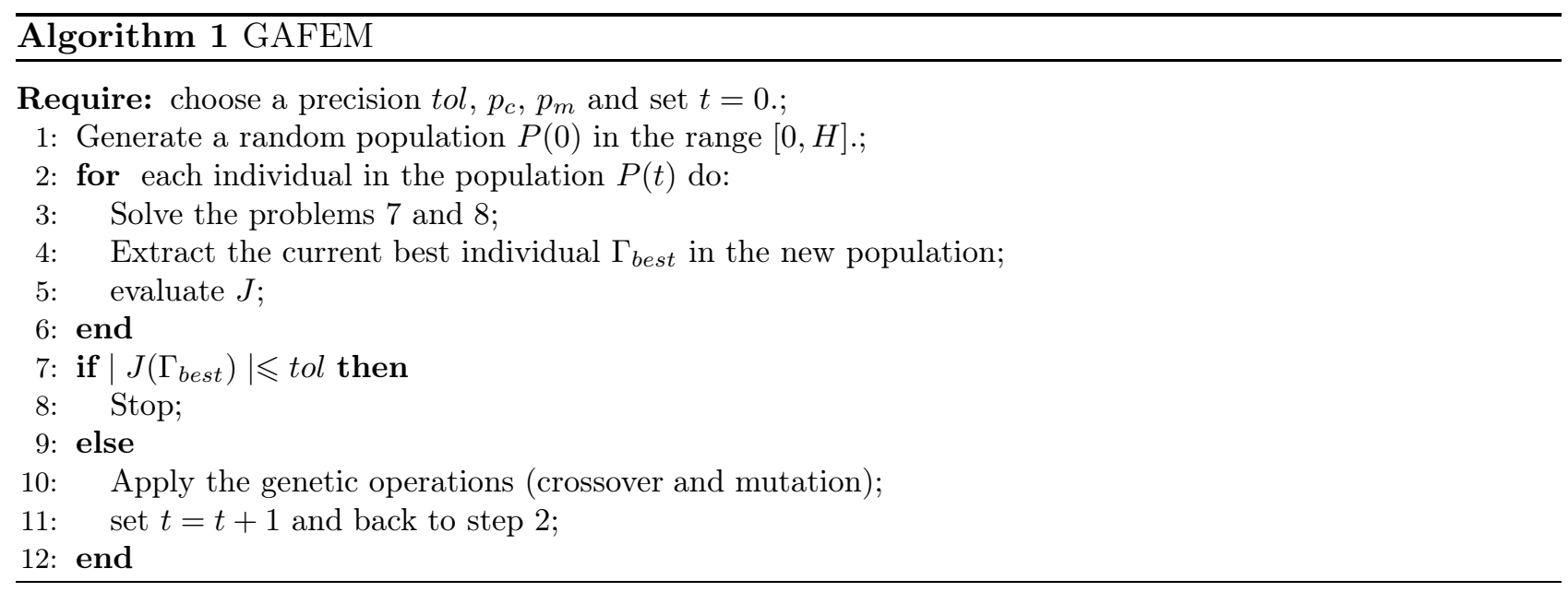

\section{Numerical results}

We assume the device domain of the MOSFET is $\Omega=[0,1] \times\left[0, \frac{1}{2}\right]$. To solve the stated problem we call the $\mathbb{P}_{1}$ finite element, for the mesh we call the algorithm proposed by P. Persson and G. Strang [12]. 
For the genetic operations we choose the tournament selection, the barycentric crossover and the nonuniform mutation operators. To tune our GAFEM algorithm we consider the parameters given in Table 1.

Table 1. GA parameters.

\begin{tabular}{cccc}
\hline Population size & Max iterations & Crossover percentage & mutation percentage \\
\hline 30 & 500 & $75 \%$ & $5 \%$ \\
\hline
\end{tabular}

Each individual in the population is assumed to be a vector of control points of Bézier curve, which we transform by the Casteljau algorithm [13] to a continuous curve, in other word to candidate boundary. The choice of Bezier curve is due to their properties of producing a continuous and smooth curve, in addition to overcome the non-differentiable character of the free boundary.

Our numerical examples are decomposed into two, first we study the approximation when the MOSFET is in the depletion mode, then we move to the enhancement mode. In the depletion mode, the gate is unbiased. The application of a positive voltage to the drain terminal implies the current flowing through the channel. While increasing the drain voltage, the depletion region becomes more wider, as a resistance to the current flow. We can remark in Fig. 2, when we vary the drain voltage from $0.1 \mathrm{~V}, 0.4 \mathrm{~V}$ to $0.7 \mathrm{~V}$, the depletion zone is much wider, the gate voltage is fixed at $0 \mathrm{~V}$.

In the enhancement mode the gate in biased. We apply a positive voltage to the drain terminal thus the current flow through the channel. We fix the drain voltage at $+0.4 \mathrm{~V}$. In this mode, the depth of the depletion region becomes higher when we increase the gate voltage, there the depletion layer will stop the current flow. The demonstration of this mode is illustrated in Fig. 3, the depletion zone is much wider when we vary the drain voltage from $0 \mathrm{~V},-0.4 \mathrm{~V}$ to $-0.7 \mathrm{~V}$.

The obtained results with the combined method GAFEM keep the physical properties holding, that is proved by Figs. 2 and 3, where the depth of the depletion region changed by varying the applied voltages to the gate and the drain terminals, and the depth is more important in the case of the enhancement mode, where the depletion region is too large to make the current flow move slowly through the channel.

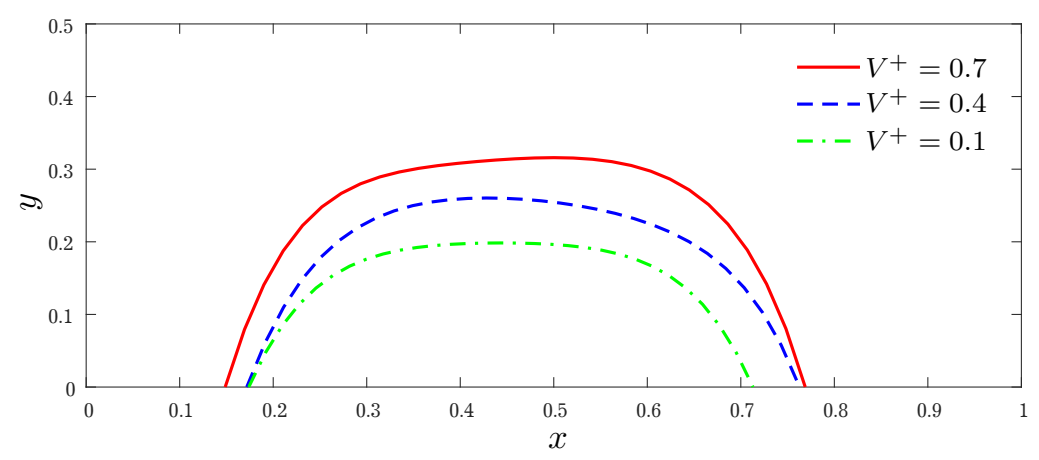

Fig. 2. Optimal boundaries under depletion mode obtained by GAFEM.

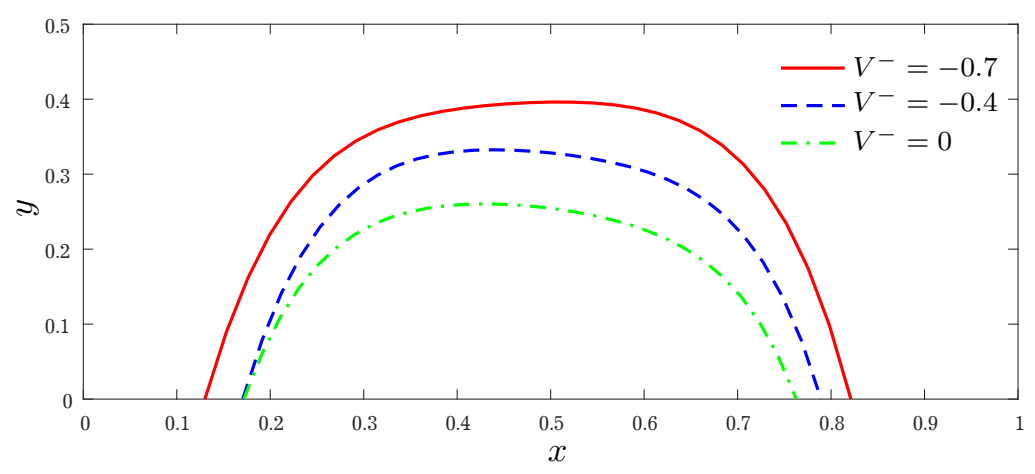

Fig. 3. Optimal boundaries under enhancement mode obtained by GAFEM.

In Table 2 we recorder the obtained optimal cost for different voltage values, for both cases, the depletion and the enhancement modes. $V^{+}$ and $V^{-}$stand for the applied voltage on the drain and the gate terminals, respectively, cost stands for the value of the cost functional $J$.

Table 2. The optimal cost for each functioning mode.

\begin{tabular}{ccc|ccc}
\hline \multicolumn{2}{c}{ Depletion mode } & \multicolumn{3}{c}{ Enhancement mode } \\
\hline$V^{+}$ & $V^{-}$ & cost & $V^{+}$ & $V^{-}$ & cost \\
\hline$+0.1 V$ & $0.0 V$ & 0.021 & $+0.4 V$ & $0.0 V$ & 0.033 \\
\hline$+0.4 V$ & $0.0 V$ & 0.043 & $+0.4 V$ & $-0.4 V$ & 0.041 \\
\hline$+0.7 V$ & $0.0 V$ & 0.078 & $+0.4 V$ & $-0.7 V$ & 0.067 \\
\hline
\end{tabular}




\section{Conclusions}

In this work, we have considered the free boundary problem for the MOSFET device. The numerical simulation has been performed using the finite element method combined with the genetic algorithm. The functioning of the MOSFET is governed by two modes: the depletion and enhancement modes, our proposed method has succeeded in the identification of the depletion layer in both modes, as it ensures their physical properties preservation, namely the depletion region increment's depth while the increment of the gate voltage.

[1] Abouchabaka J., Aboulaich R., Guennoun O., Nachaoui A., Souissi A. Shape optimization for a simulation of a semiconductor problem. Mathematics and Computers in Simulation. 56, 1-16 (2001).

[2] Friedman A. A. Free Boundary Problems Associated with Multiscale Tumor Models. Mathematical Modeling of Natural Phenomena. 4 (3), 134-155 (2009).

[3] Ellabib A., Nachaoui A. On the numerical solution of a free boundary identification problem. Inverse Problems in Engineering. 9, 235-260 (2001).

[4] Sze S. M. Semiconductor Devices: Physics and Technology. John Wiley and Sons, New Jersey (1985).

[5] Zhang R., Sun J. The reconstruction of obstacles in a waveguide using finite elements. Journal of Computational Mathematics. 36, 29-46 (2017).

[6] Ciarlet P. G. The Finite Element Method for Elliptic Problems. Studies in mathematics and its applications. North Holland (1978).

[7] Goldberg D. E. Genetic Algorithm in search, optimisation, and machine learning. Addison-Wesley, Reading, MA (1989).

[8] Pironneau O. Optimal Shape Design for Elliptic Systems. In: Drenick R. F., Kozin F. (eds) System Modeling and Optimization. Lecture Notes in Control and Information Sciences, vol.38. Springer, Berlin, Heidelberg, p. 42-66 (2005).

[9] Chakib A., Nachaoui A., Nachaoui M. Existence analysis of an optimal shape design problem with non coercive state equation. Nonlinear Analysis: Real World Applications. 28, 171-183 (2016).

[10] Holland J. Adaptation in natural and artificial systems: an introductory analysis with applications to biology, control, and artificial intelligence. MIT press (1975).

[11] Prautzsch H., Boehm W., Paluszny M. Bézier and B-Spline Techniques. Springer-Verlag, Berlin, Heidelberg (2002).

[12] Persson P. O., Strang G. A Simple Mesh Generator in MATLAB. SIAM Review. 46, 329-345 (2004).

[13] Demengel G., Pouget J. P. Modèles de Béziers, de B-splines et de NURBS: Mathématiques des courbes et des surfaces. Ellipses (1998). 


\title{
Реконструкція збідненого шару в МДП-структурі за допомогою генетичних алгоритмів
}

\author{
Ель Язиді Юнес, Еллабіб Абделлатиф \\ Університет Каді Айяд, \\ Лабораторія прикладної математики та обчислювальної техніки, \\ Факультет науки і техніки, \\ Авеню Абделькрим Ель-Хаттабі Б. П., 549, Марракеш, Марокко
}

\begin{abstract}
У цій роботі розглядається напівпровідниковий пристрій на основі МПД-структури. Густину носіїв заряду в МПД-структурі змодельовано рівнянням дрейфової дифузії. Для того, щоб отримати просте рівняння Лапласа або Пуассона, використано формули густини заряду за умов рівноваги. Означено функціонал витрат для формулювання задачі оптимізації форми. Доведено існування оптимального розв'язку. Для розв'язання задачі оптимізації розроблено числовий підхід на основі методу скінченних елементів у поєднанні з генетичним алгоритмом. Для підтвердження обгрунтованості запропонованого підходу наведено декілька чисельних прикладів.
\end{abstract}

Ключові слова: напівпровідник, оптимізація форми, скінченний елемент, генетичний алгоритм. 